\title{
Kombinierte Sozio- und Psychotherapie bei demenzbetroffenen Paaren: Fallberichte aus der DYADEM-Studie
}

\section{Combined Socio- and Psychotherapeutic Intervention in Couples Affected by Incipient Dementia: Case Reports from the DYADEM Study}

Autoren

Institute
Andreas Häusler ${ }^{1}$, Kerstin Krause-Köhler ${ }^{2}$, Eszter da Conceicao ${ }^{2}$, Mechthild Niemann-Mirmehdi ${ }^{2}$, Michael Rapp ${ }^{2}$, Johanna Nordheim ${ }^{3}$

${ }^{1}$ Sozial- und Präventivmedizin, Universität Potsdam

${ }^{2}$ Klinik für Psychiatrie und Psychotherapie im St. Hedwig Krankenhaus, Charité Berlin

${ }^{3}$ Insitut für Medizinische Soziologie, Charité Universitätsmedizin Berlin

\section{Schlüsselwörter \\ - Demenz \\ - Soziotherapie \\ - Psychotherapie \\ - Fallvignetten \\ Intervention \\ Keywords \\ - dementia \\ - sociotherapy \\ - psychotherapy \\ - case report \\ - intervention}

eingereicht 3. Dezember 2013 akzeptiert 3. Dezember 2014

Bibliografie

DOI http://dx.doi.org/

10.1055/s-0034-1396838

Online-Publikation: 9.2.2015

Psychother Psych Med 2015;

65: 238-242

(c) Georg Thieme Verlag KG

Stuttgart - New York

ISSN 0937-2032

Korrespondenzadresse

Andreas Häusler

Universität Potsdam

Sozial- und Präventivmedizin

Am Neuen Palais 10

14469 Potsdam

Andreas.Haeusler@Uni-

Potsdam.de

\section{Zusammenfassung}

$\nabla$

Wir stellen eine kombinierte sozio- und psychotherapeutische Intervention bei von beginnender Demenzerkrankung betroffenen Paaren vor. Dabei zeigen wir die Bedeutung der Berücksichtigung sozialer und psychologischer Ressourcen im Therapieprozess im Einzelfall. Zu 2 ergänzenden Fallberichten zeigen wir, dass diese Prinzipien und Interventionen der Sozio- und Psychotherapie bei beginnender Demenzerkrankung erfolgreich angewendet werden können.

\section{Einleitung}

Verschiedene psychosoziale Interventionen bei Demenzkranken oder ihren Angehörigen konnten Effekte auf Lebensqualität, Pflegekompetenz, Kognition, Alltagsaktivitäten und insbesondere verzögerte Heimeinweisung zeigen [1]. Obgleich einige Interventionen psychotherapeutische Aspekte beinhalten [1], sind spezifische psychotherapeutische Studien bei Demenz selten und kaum auf das Paar Betroffener/Angehöriger fokussiert; oft werden hier kognitive und psychosoziale Interventionen kombiniert [1-3].

Die DYADEM (Dyadisches Coping bei Demenz)Studie untersucht ein Trainings- und Beratungsprogramm, das Menschen mit beginnender bis mittelschwerer Demenz und ihren Partnern dabei helfen soll, persönliche und gemeinsame Bewältigungsfähigkeiten zu stärken.

Während u.a. in der S3-Leitlinie Demenz [4] sowohl psychosoziale Interventionen für die $\mathrm{Er}$ krankten als auch Angehörigenberatung empfohlen werden, wollte das Forschungsvorhaben die Frage beantworten, ob eine kombinierte Intervention für Paare effektiver Ressourcen fördern, Autonomie und Lebensqualität erhalten kann als eine Standardberatung (Krankheitsaufklärung, nichtmedikamentöse Therapiemöglichkeiten, soziale

\section{Abstract \\ $\nabla$}

We present a combined socio- and psychotherapeutic intervention in couples affected by incipient dementia. We show that the consideration of both social and psychological resources play an important role in the therapy process. 2 additional case reports suggest that those principles of both socio- and psychotherapy can be successfully applied in early dementia.
Hilfen usw.) wie sie z.B. in einigen Gedächtnissprechstunden angeboten wird.

Das im Rahmen der Studie entwickelte Unterstützungsprogramm beinhaltet bewährte Methoden der Verhaltenstherapie sowie der systemischen Beratung [5].

Die standardisierten therapeutischen Module ( $\bullet$ Abb. 1) wurden im Einzelnen so individualisiert, dass der Demenz-Patient nicht überfordert wurde und sie der spezifischen Paardynamik gerecht wurden.

Die Therapeuten sollten eine „allparteiliche“ und nicht wertende Haltung einnehmen und dabei die Kontextabhängigkeit von Problemen im Blick behalten.

Im Vordergrund stand die lösungsfokussierte Arbeit, wobei die Therapeuten als Rollenvorbild fungierten (z. B. klare, transparente Kommunikation). Darüber hinaus benötigt die Arbeit mit Demenzerkrankten weitgehende Flexibilität, Beobachtungs- und Reflexionsvermögen hinsichtlich spezieller Herausforderungen (wie Tagesform des Patienten, akute Krisen, weitere Erkrankungen oder Pflegebedarf). 


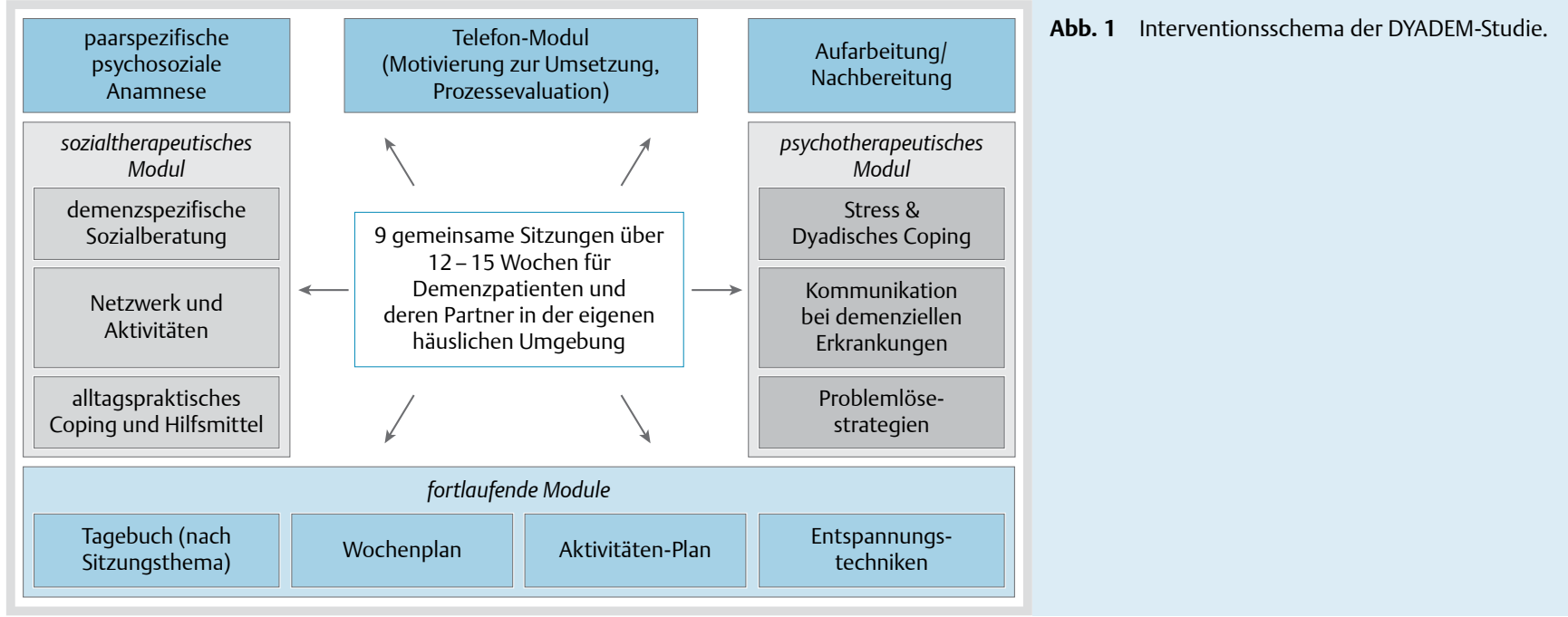

\section{Paarspezifisches psychosoziales Unterstützungs- programm \\ $\nabla$}

Das Angebot erfolgte im eigenen Zuhause und umfasste 9 Sitzungen (davon 2 telefonisch), die 1-2-wöchentlich durchgeführt wurden. Die etwa 2-stündigen Termine enthielten neben psychound soziotherapeutischen Schwerpunkten auch fortlaufende Module, die einerseits die Intervention strukturieren andererseits auch als Bindeglied zwischen den einzelnen Inhalten fungieren sollten. Der trialogischen Grundidee folgend wurde die Intervention stets mit beiden Partnern gemeinsam durchgeführt. Abgesehen vom Erstgespräch (1. Sitzung) wurden die einzelnen Sitzungen je nach ihrer inhaltlichen Ausrichtung entweder von einem Sozial- oder Psychotherapeuten durchgeführt. Folgend werden die einzelnen Therapiebausteine kurz umrissen.

\section{Paarspezifische Anamnese}

Die paarspezifische Anamnese hat eine erste gemeinsame Exploration des Paares zum Ziel. Dabei wird die Ausgangsbasis des Paares, d.h. die Lebens- und Beziehungssituation, aktuelle Belastungen, Veränderungswünsche, Erwartungen an die Therapeuten usw., erhoben. Ergänzend erfolgt zudem eine ausführliche Krankheitsaufklärung.

\section{Sozialtherapeutisches Modul}

Die 3 sozialtherapeutischen Sitzungen verfolgen verschiedene Schwerpunkte. Neben einer demenzspezifischen Sozialberatung zu Themen wie Vorsorgevollmacht, Pflegeversicherung, Schwerbehinderung u.a. rückt das Aktivitätenprofil des Paares in den Fokus. Es wird hierbei gemeinsam mit dem Paar an einer Aktivierung gearbeitet, gleichzeitig wird aber auch möglichen Überlastungen vorgebeugt. Daneben dient die Erstellung einer „Netzwerkkarte" der Visualisierung und ggf. Erweiterung des sozialen Netzwerks. Ein weiterer Schwerpunkt ist das alltagspraktische Coping.

Zudem steht das Thema Hilfsmittel im Fokus: mit der Erarbeitung individueller demenzspezifischer Erinnerungshilfen sowie alltagspraktischer Hilfen im Wohnumfeld soll eine Erweiterung der Handlungskompetenzen erreicht werden.

\section{Psychotherapeutisches Modul}

Die psychotherapeutischen Sitzungen fokussieren zunächst das Thema Stress. Im Anschluss an eine Psychoedukation werden in- dividuelle, aber auch partnerschaftliche Copingstrategien thematisiert. Durch zirkuläres Fragen soll dabei ein Verständnis für die Perspektive des Partners entwickelt werden. Die Inhalte können zusätzlich durch Rollenspiele gefestigt werden. Inhalt der 2 . Sitzung ist eine demenzspezifische Kommunikation, die sich gerade auch an den Erfordernissen und Möglichkeiten des Betroffenen ausrichtet. Komplettiert wird der psychotherapeutische Interventionsteil durch eine Einführung in das Problemlösetraining [6].

\section{Fortlaufende Module}

Das hier vorgestellte Unterstützungsprogramm beinhaltet sitzungsübergreifende Einheiten: So dienen ein „Tagebuch“ und ein Wochenplan der Strukturierung des Alltags und dem Aufbau positiver Aktivitäten. Darüber hinaus werden zur Stressreduktion die Teilnehmer in 2 Entspannungsverfahren unterrichtet. 2 flankierende Telefonmodule dienen der Festigung der Inhalte sowie der Prozessevaluation.

Individuell ausgerichtet erfolgen Empfehlungen für die Inanspruchnahme weiterführender Versorgungs- und Unterstützungsangebote (z.B. Begegnungsstätten, Selbsthilfegruppen, ambulante Psychotherapie). Eine systematische Überleitung im Sinne eines Case-Managements ist hingegen nicht Ziel des Behandlungsprogramms.

\section{Auf- und Nachbereitung}

Die letzte Sitzung hat die allgemeine Konsolidierung der erarbeiteten Inhalte zum Ziel. Dies erfolgt mithilfe einer „Time-Line mit Ankern“ [7]: Die individuellen Ergebnisse der Intervention werden resümierend mithilfe von Schnüren und Karten („Ankern“) grafisch dargestellt und wichtige Erkenntnisse („Meilensteine“) werden dabei besonders gewichtet. Abschließend wird gemeinsam mit dem Paar eine positive Zukunftsvision entwickelt und ihre Ressourcen hierbei nochmals rückgemeldet.

\section{Die DYADEM-Studie}

Die DYADEM-Studie wurde als BMBF-gefördertes Teilprojekt im Rahmen des Forschungsverbundes „Autonomie trotz Multimorbidität im Alter (AMA)“ von 2011-2013 als randomisierte, kontrollierte Interventionsstudie in Berlin mit 164 Personen durchgeführt. Es wurden Paare eingeschlossen, die gemeinsam im privaten Haushalt wohnten. Zudem musste bei dem erkrankten Partner eine fachärztliche Demenzdiagnose vorliegen. Aus- 


\begin{tabular}{|c|c|c|}
\hline Primäres Outcome & Autonomie & $\begin{array}{l}\text { - ADL/IADL (Activities of Daily Living/Instrumental Activities of Daily Living) } \\
\text { - OPSA (Older Adults Overprotection Scale) } \\
\text { - FKpA (Kompetenzüberzeugung pflegender Angehöriger) }\end{array}$ \\
\hline \multirow[t]{2}{*}{ Sekundäres Outcome } & Lebensqualität & $\begin{array}{l}\text { - WHOQL-BREF (WHO Quality-of-Life Scale) } \\
\text { - QOL-AD (Quality of Life in Alzheimer's Disease) }\end{array}$ \\
\hline & Zeit bis zur Einweisung ins Pflegeheim & \\
\hline Moderatoren & Ressourcen & $\begin{array}{l}\text { - DCI (Dyadic Coping Inventory) } \\
\text { - F-SOZU K-14 (Fragebogen zur Sozialen Unterstützung) } \\
\text { - Global Measure of Equity/Inequity }\end{array}$ \\
\hline \multirow[t]{2}{*}{ Prädiktoren } & Demenz/Begleitsymptome & $\begin{array}{l}\text { - MMST (Mini Mental Status Test) } \\
\text { - GDS (Global Deterioration Scale) } \\
\text { - NPI (Neuropsychiatrisches Inventar) }\end{array}$ \\
\hline & Depression & $\begin{array}{l}\text { - GDS-15 (Geriatrische Depressionsskala) } \\
\text { - PSS (Perceived Stress Scale) }\end{array}$ \\
\hline
\end{tabular}

schlusskriterien waren ein MMST-Wert $<15$ Punkte, eine Demenzerkrankung beider Partner sowie das Vorliegen weiterer psychischer Diagnosen (F10-29, F32.2 und F32.3), die Teilnahme an Medikamentenstudien mit nicht zugelassenen Wirkstoffen oder gezielten Verhaltensinterventionen. $\bullet$ Tab. 1 zeigt die Zielkriterien und entsprechenden Erhebungsinstrumente.

\section{Fallberichte}

Im Folgenden werden exemplarisch, qualitativ für unterschiedliche Problemlagen betroffener Paare und für das Vorgehen innerhalb des Unterstützungsprogramms, 2 Fälle vorgestellt. Die beschriebenen Personen wurden anonymisiert und ihre wesentlichen Angaben verändert. Die medizinisch-neuropsychologischen Basisdaten entstammen der CERAD-Plus-Testung sowie der ärztlichen Anamnese aus den konsultierten Gedächtnissprechstunden.

\section{Fall 1: Sozialer Rückzug bei einer Alzheimer-Demenz}

Das Ehepaar F. ist seit 45 Jahren verheiratet, hat 2 Kinder und lebt gut situiert. Ein Jahr zuvor wurde bei Herrn F. eine Alzheimer-Demenz diagnostiziert, seine Frau leidet seit einigen Jahren an Rückenschmerzen.

Ehepaar F. lebt in einer Eigentumswohnung in direkter Nachbarschaft zur Tochter und ist aktiv in die Betreuung der beiden Enkelkinder eingebunden. Als ehemaliger Schuldirektor vermochte es Herr F. bisher, alle administrativen Aufgaben am PC zu erledigen. Auch die Gartenpflege und die Instandhaltung des Hauses oblagen seiner Verantwortung. Seine Freizeit gestaltete er aktiv mit Thai-Chi, dem Sammeln von Filmen und Lesen.

Im Verlauf des letzten Jahres vermied Herr F. immer mehr gemeinsame Aktivitäten und gab viele frühere Hobbys auf. Dies wurde primär von seiner Frau als zentraler Verlust an Lebensqualität und Freude erlebt und führte beim Paar zunehmend zu Konflikten.

\section{Medizinisch-neuropsychologische Basisdaten}

Vor 6 Jahren wurde Herrn F. wegen Darmkrebs chirurgisch behandelt. Die testpsychologisch festgestellten Defizite mit Alltagsrelevanz sind in erster Linie in den Bereichen Neugedächtnisbildung, semantisches Altgedächtnis, sowie der exekutiven Funktionen zu finden. Bei der Diagnosestellung erreichte Herr F. einen MMST-Wert von 25 Punkten, ein Jahr später 24 Punkte und nach 18 Monaten 21 Punkten. Die geriatrische Depressionsskala (GDS) war unauffällig. Die Beziehung selbst wird von beiden Partnern als ausgeglichen bewertet.

\section{Interventionsverlauf}

Zu Beginn der Erkrankung konnte das Ehepaar auf die kritische Lebenserfahrung mit der Krebserkrankung des Betroffenen zurückgreifen. Trotz dieser guten Copingstrategien führten aber zunehmende Funktionseinschränkungen bei Herrn F. bald zu einem zunehmenden Rückzug von der aktiven Teilhabe am sozialen Leben und zur Aufgabe von Tätigkeiten im häuslichen Bereich. Dadurch geriet die Ehefrau in eine Überforderungssituation, da ihr Mann sie nicht mehr in gewohntem Maße unterstützte. Die Therapeuten näherten sich dieser Problemkonstellation auf verschiedenen Ebenen:

Bereiche stärken. Nach einer anfänglichen Bestandsaufnahme wurde deutlich, dass viele Ressourcen noch bestanden und neu aktiviert werden konnten. Hinsichtlich des familiären Netzwerks wurde besprochen, Hilfsangebote der Tochter im Sinne einer Generationenbilanz verstärkt anzunehmen. Da die Betreuung der Enkel einen zufriedenstellenden Bereich darstellte, der gestärkt und erhalten werden sollte, zielte die Intervention darauf ab, einen transparenten Umgang mit der Erkrankung gegenüber den Enkeln zu fördern. Dies wurde mithilfe kindgerechter Literatur zum Thema Demenz erfolgreich angeregt.

Autonomie wahren. Ein weiterer Aspekt der Rückzugtendenzen von Herrn F. waren Orientierungsprobleme, bspw. bei Spaziergängen im Wohnumfeld. Er war hier sehr reflektiert und berichtete offen über seine Ängste und Ausfälle. Durch die Verkleinerung seines Aktionsradius büßte er deutlich an Selbstständigkeit ein. Verschiedene alltagspraktische Strategien bei Orientierungsverlust wurden mit ihm erörtert. Herr F. entschied sich zur Stärkung seines Sicherheitsgefühls bei Spaziergängen, ein 3-Tasten-Handy und einen Umfeld-Stadtplan zu nutzen. Um unabhängig von der Ehefrau wieder an einer Gruppe eigenständig teilnehmen zu können, wurde ein Mobilitätshilfedienst eingebunden. Das Interventionsziel einer größtmöglichen Autonomie wurde hier auch von der Ehefrau mitgetragen und unterstützt. Entspannen. Mithilfe der Progressiven Muskelrelaxation (PMR) wurden Phasen der Entspannung gegenüber Phasen der Aktivität kontrastiert. Als Nebeneffekt konnte erreicht werden, dass eine aktiv gestaltete Tagesstruktur die Rückzugstendenzen durch bewusst geplante Phasen der Entspannung ersetzte. Beide Partner empfanden die PMR als positiv und führten diese auch weiterhin gemeinsam mithilfe einer CD durch.

Den Überblick behalten. Die Intervention im häuslichen Kontext ermöglichte es konkrete Strategien zu entwickeln, sodass der Betroffene wieder mehr Aufgaben zu Hause übernehmen bzw. in modifizierter Form beibehalten konnte. Für Herrn F. wurde zunächst ein übersichtlicher Aufgaben- und Terminplan erstellt, der die lose Zettelsammlung ersetzte und ihm so er- 
möglichte, den Überblick zu behalten. Des Weiteren zeigte sich, dass viele alltagspraktische Schwierigkeiten darauf beruhten, dass Herr F. die Aufbewahrungsorte für wichtige Papiere, Werkzeuge oder Büromaterialien nicht erinnerte. Als Hilfsstrategien wurden ein Merkheft und individuell gestaltete Symbolkarten an Schranktüren bzw. Schubladen eingesetzt. So war es ihm möglich, vorerst seine bisherige Rolle als Verwalter des Haushaltes weiter auszufüllen. Für das eigenständige und partnerschaftliche Krankheitsmanagement erwies sich die Einführung des „LOGBUCH Demenz [8]“ als gutes Hilfsmittel.

\section{Ergebnisse}

Durch die Intervention konnte erreicht werden, dass sich die Rückzugstendenzen von Herrn F. nicht weiter verstärkten. Vielmehr entwickelte er eine neue Motivation, sich in soziale Beziehungen und die Partnerschaft einzubringen. Es gelang, gemeinsam mit beiden Partnern eine adaptive Zukunftsperspektive zu entwickeln und das Selbstwirksamkeitsgefühl von Herrn F. zu stärken. Insgesamt wurde dem Paar der Zusammenhang zwischen seinem Rückzug und ihrer Verantwortungsübernahme und dem daraus resultierenden Überlastungserleben verdeutlicht.

\section{Fall 2: Überforderung im Anpassungsprozess bei einer gemischten Alzheimer-Demenz}

Das seit 5 Jahren verheiratete Paar Z . kennt sich beruflich bereits seit 30 Jahren, während beide damals noch in jeweils kinderloser erster Ehe lebten. Herr Z. arbeitete als Arzt, Frau Z. als Forschungsassistentin. Nach der Lösung aus ihren damaligen Beziehungen vor 8 Jahren kamen sich beide nun auch privat näher. Im Jahr darauf entschieden sie sich für eine gleichzeitige Berentung, zogen zusammen und heirateten.

Den neuen Freiraum genossen beide intensiv durch gemeinsame Aktivitäten wie Hobbies, Ausflüge und Reisen. Herr Z. verpflichtete sich zudem, den Hof des Wohnhauses zu bepflanzen und zu pflegen. Zuvor hatte er noch bei seinem Tennisverein ehrenamtlich die Buchführung übernommen.

Bald jedoch verschlechterte sich seine Konzentrationsfähigkeit: selbst einfache Rechenaufgaben wurden für ihn zur Herausforderung. Frau Z. kompensierte anfangs die Defizite ihres Mannes und übernahm dessen Aufgaben zusätzlich, wenn sie einschätzte, dass dieser überfordert war.

Die Diagnosestellung einer gemischten Alzheimer-Demenz bei Herrn Z. erfolgte vor 1,5 Jahren. Zunehmend erlebte sich Herr Z. überfordert und neigte insbesondere in Gesprächen zu Rückzugstendenzen. Frau Z. reagierte auf diese Veränderungen mit Rastlosigkeit, Besorgnis und Anspannung, jedoch immer auch mit großem Engagement, den Erhalt seiner Gesundheit zu begünstigen.

\section{Medizinisch-neuropsychologische Basisdaten}

Ein vom Neurologen festgestelltes extrapyramidalmotorisches Syndrom führte zunächst bei Herrn Z. zu der Verdachtsdiagnose einer Parkinson-Demenz.

Die neuropsychologische Testung ergab eine deutliche kognitive und psychomotorische Verlangsamung und eine Schwäche des Altgedächtnisses sowie eine materialabhängige Störung der Neugedächtnisbildung. Der MMST erwies sich bei Herrn Z. als relativ stabil (21 Punkte). Die GDS-Werte stiegen über 12 Monate von 1 auf 8 . Auch bei der Ehefrau erhöhte sich der GDS-Wert von 3 auf 10. Somit zeigten beide im Zeitverlauf eine depressive Symptomatik. Die Beziehung beurteile das Paar als relativ ausgeglichen.
Interventionsverlauf

Kommunikationsbasis schaffen. Bereits zu Beginn der Intervention wurden Herrn Z.s Schwierigkeiten deutlich, sich sprachlich zu äußern. Frau Z. sprang hier oft ein und übernahm es, für ihn zu antworten. Dieses Ungleichgewicht wurde verdeutlicht und durch klare Sprachregeln wurde eine gemeinsame Kommunikationsbasis geschaffen. Dies führte zu einer aktiveren Gesprächsbeteiligung von Herrn Z. Von den Therapeuten wurden beide Partner abwechselnd angesprochen und involviert. Stresspegel senken. Während des Interventionsschwerpunktes „Stressbewältigung und Entspannung“ verdeutlichten die Therapeuten den aktuellen Stresspegel des Paares und den positiven Effekt entspannender Tätigkeiten. Hier stellte sich heraus, dass die Gartenarbeit entspannend auf Herrn Z. wirkte, er sich jedoch dabei mitunter selbst überforderte. Das Paar vereinbarte, in solchen Fällen die Arbeiten gemeinsam und mit Pausen zu erledigen.

Soziale Kontakte reaktivieren. Herr Z. verfügt über ein tragfähiges Netzwerk, während bei Frau Z. eine gering familiäre Einbettung und wenig freundschaftliche Kontakte deutlich wurden. Ihre Aktivitäten nahm Frau Z. überwiegend mit ihrem Partner wahr. Hier zeigten die Therapeuten die Bedeutung eigener Aktivitäten und Interessen auf sowie Möglichkeiten, in denen Herr Z. allein zu Hause bleiben konnte. Die Reaktivierung eigener Kontakte stellte sich bei Frau Z. als Chance für mehr Lebensfreude dar. Anhand der erstellten Netzwerkkarten wurde zudem der Bedarf externer Hilfen verdeutlicht, um einer Überforderung und Vereinsamung vorzubeugen.

\section{Ergebnisse}

Das Paar Z. wurde in die Lage versetzt, das Maß ihrer Aktivitäten an ihre Ansprüche und ihr Befinden anzupassen, um so Überund Unterforderungen entgegenzuwirken. Durch den Aufbau regelmäßiger Rituale konnte die partnerschaftliche Absprachefähigkeit erhöht werden. Eine Anbindung an ein professionelles Helfersystem konnte zudem etabliert werden. Auch erlebte sich Herr Z. wieder selbstwirksamer und beteiligte sich verstärkt am sozialen Leben. Frau Z. wiederum erkannte für sie nicht hilfreiche eigene Denkmuster und konnte sich auf neue Weise mit der Krankheitsbewältigung auseinandersetzen.

\section{Fazit für die Praxis}

Bei beginnenden Demenzen zeigen sich vielfältige Symptome von Überlastung und Trauer, die in der spezifischen Konstellation eines Paares manifest werden.

Die vorliegenden Fälle machen deutlich, dass eine individualisierte Intervention es ermöglicht, verhaltenstherapeutische und soziotherapeutische Angebote auf der Grundlage neuropsychologischer Erkenntnisse mit den Betroffenen vor Ort umzusetzen und zu evaluieren. Während beim 1. Fall konkrete soziotherapeutische Unterstützungsmaßnahmen und Verhaltenspläne zu einem Zugewinn an Autonomie führten, zeigte sich im 2. Fall durch die Lösung kommunikativer Defizite erst die Möglichkeit zur Intervention. Erst daran anschließend konnten konkrete Angebote zur Wiederaufnahme von Kontakten bei der nicht betroffenen Partnerin erkannt und gemeinsam umgesetzt werden.

Hervorzuhebende Aspekte dieser Intervention sind der häusliche Zugang und damit die Einbeziehung der Kontextbedingungen. Einerseits werden so verbreiteten Mobilitätsproblemen 
der häufig hochaltrigen Klientel sowie zusätzlichen Stressoren wie die Einhaltung wöchentlicher Außentermine vorgebeugt. Andererseits kann durch den Hausbesuch eine individuelle Wohnungs- und Hilfsmittelberatung erfolgen. Schließlich kann davon ausgegangen werden, dass der Demenzbetroffene sich in seinem Lebensumfeld sicherer fühlt und damit seine Therapiefähigkeit erhöht wird. Darüber hinaus erscheint die in den einzelnen Modulen enthaltene Vielfalt an Psychoedukation und Interventionsmethoden als sinnvolle Grundlage, demenzbetroffene Paare individuell in ihrer Problemlage abzuholen und ihnen somit das direkte Erleben von Unterstützung und Selbstwirksamkeit zu ermöglichen.

Für die Zukunft wäre denkbar, dass sich das Angebot als frühzeitiger Baustein in einer demenzspezifischen Versorgungskette etablieren lässt und so auch eine längerfristige Betreuung von demenzbetroffenen Paaren gewährleistet wäre.

Interessenkonflikt: Die Autoren geben an, dass keine Interessenkonflikte bestehen.

\section{Literatur}

1 Olazarán J, Reisberg B, Clare L et al. Nonpharmacological therapies in Alzheimer's disease: a systematic review of efficacy. Dement Geriatr Cogn Disord 2010; 30: 161-178

2 Kurz A, Thöne-Otto A, Cramer B et al. CORDIAL: cognitive rehabilitation and cognitive-behavioral treatment for early dementia in Alzheimer disease: a multicenter, randomized, controlled trial. Alzheimer Dis Assoc Disord 2012; 26: 246-253

3 Gitlin LN, Winter L, Dennis MP et al. A biobehavioral home-based intervention and the well-being of patients with dementia and their caregivers: the COPE randomized trial. JAMA 2010; 304: 983-991

4 Deutsche Gesellschaft für Psychiatrie, Psychotherapie und Nervenheilkunde (DGPPN), Deutsche Gesellschaft für Neurologie, Hrsg. Diagnoseund Behandlungsleitlinie Demenz. Reihe: Interdisziplinäre S3-Praxisleitlinien. Berlin, Heidelberg: Springer 2010

5 Häusler A, Krause-Köhler K, Niemann-Mirmehdi M et al. Psychosoziale Therapie bei beginnender Demenz. Das DYADEM-Unterstützungsprogramm für Menschen mit Demenz und ihre Partner. Frankfurt: Mabuse 2014

6 D'Zurilla TJ, Goldfried MR. Problem solving and behavior modification. J Abnorm Psychol 1971; 78: 107-126

7 Theuretzbacher K, Nemetschek P. Coaching und Systemische Supervision mit Herz, Hand und Verstand: Handlungsorientiert arbeiten, Systeme aufbauen. Stuttgart: Klett-Cotta 2013

8 von der Ahe HE. LOGBUCH Demenz. Schnittstellenmanagement und Angehörigen-Empowerment in der Betreuung von Menschen mit Demenz. Pro Alter 2011; 43: 43-46 